\title{
Depth of Central Venous Catheterization by Intracardiac ECG in Paediatric Patients
}

\author{
Prerana N. Shah", b, Jithesh Appukutty ${ }^{\mathrm{a}}$, Deepa Kane ${ }^{\mathrm{a}}$
}

\begin{abstract}
Background: Central venous catheterization is a frequent procedure in cardiac surgery and intensive care setting. Its faulty positioning can cause severe complications, which can be fatal and erroneous central venous pressure measurement.
\end{abstract}

Methods: In this prospective study, the right internal jugular vein (IJV) was cannulated in cardiac surgery patients over a period of four months and catheter tip positioning was guided by means of intracardiac ECG. The insertion depth was registered at the position of maximum P-wave amplitude and the catheter was fixed after withdrawing a further two cm. Pearson's correlation coefficient was calculated to categorize any relation between height, body weight or body surface area (BSA) and the depth of insertion required to locate correct positioning. Plots of distance versus patient's height were made, and regression lines and equations were calculated. Bland-Altman analysis of data was done to compare the old formulae with our derived formulae. 155 adult patients were studied.

Results: Distances measured were found to be highly correlating with patient's height, followed by body surface area (BSA) and weight. For right IJV cannulation in valvular surgeries in adults, the depth of insertion (cm) was (Height in $\mathrm{cm} / 15)+2$ and in nonvalvular surgeries in adults, it was (Height in $\mathrm{cm} / 15)+1.4$.

Conclusions: The devised formulae accurately predicted the required depth of catheters thereby reducing the possibility of complications and need for radiographic confirmation.

Keywords: Heart; Catheterization; Monitoring; Intracardiac electrocardiography; Veins; Jugular

\footnotetext{
Manuscript accepted for publication September 1, 2011

a Department of Anaesthesiology, Seth GSMC \& KEM Hospital, Parel, Mumbai 400012, India

${ }^{b}$ Corresponding author: Prerana N. Shah. 73 B, Varma Villa, Vitthalbhai Road, Vile Parle West, Mumbai 400056, Maharashtra, India. Email: pps@kem.edu
}

doi:10.4021/ijcp17e

\section{Introduction}

Placement of a central venous catheter or central venous catheterization $(\mathrm{CVC})$ is a frequent procedure during cardiac anaesthesia and intensive care. Its malposition can cause faulty and erroneous central venous pressure measurement along with several fatal complications such as thrombosis of the great vessels, arrhythmias, cardiac perforation and cardiac tamponade. To avoid these fatal complications, the CVC is recommended to be placed in the distal superior vena cava (SVC) i.e., outside of the cardiac chamber. Malposition related complications could be serious. Thus, to suggest a guideline for the proper length of a CVC inserted through the internal jugular vein (IJV), we measured the distance from the skin puncture site to the SVC-right atrium (RA) junction by using intracardiac lead ECG monitoring. The formulae available for the depth in pediatric patients as by Andropoulos is that when height of the patient up to height $(\mathrm{H})$ of 100 $\mathrm{cm}$, the formula is $\mathrm{H} / 10-1$, and when the height is more than $100 \mathrm{~cm}$ it is $\mathrm{H} / 10$ - 2 [1]. However, we did not get at those values. Hence, the need for the study was decided over a period of four months so that after the analysis of our data, we could co-relate and decide whether the available formulae were acceptable. The aim of our study was to study the average depth of insertion of central venous catheter and to try to formulate a general guideline for depth of CVC through the right IJV.

\section{Methods}

Ethical approval for this study was taken. A valid informed consent was obtained. The patients up to 12 years of age were selected according to the inclusion criteria, those undergoing cardiac surgery and requiring central venous catheterization and the exclusion criteria being consent refusal or any abnormal ECG rhythm respectively. The technique used for CVC was the Seldinger technique in all patients. Constant and standard approach used for IJV catheterization was anterior approach (skin puncture at the apex of the triangle formed by the two heads of sternocleidomastoid muscle). CVC was 
Table 1. Demographic Data

\begin{tabular}{lllll}
\hline & & Male & Female & Total \\
\hline \multirow{2}{*}{ Paediatric } & Height $<100$ & 28 & 16 & 44 \\
& Height $>100$ & 19 & 6 & 25 \\
Total & 47 & 22 & 69 \\
\hline
\end{tabular}

performed after the induction of general anesthesia using standard institutional protocol for general anaesthesia. The CVC set included the standard multi lumen central venous catheter and a connection cable with a clip for connecting

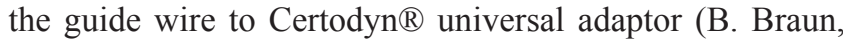
Melsungen, Germany). Once the IJV was identified, the sterile connection cable was clamped to the guide wire in order to connect it to the Certodyn $\AA$ universal adaptor, which allowed the operator to switch from a surface to an intracardiac ECG, the guide wire acting as a unipolar electrode by using a switch. The CVC was advanced together with the guide wire until the rise in $\mathrm{P}$ wave amplitude remained the same. The lead cable coming from the monitor has to be fixed to the Universal adaptor, which has a cable with clamp and has to be fixed to the red skin electrode. During advancement of the catheter with guide wire, the disposable lead cable has to be clamped to the wire and the Universal adaptor. Depth of insertion can be known from the markings on the catheter. The guide wire was removed and the central venous catheter was withdrawn by $2 \mathrm{~cm}$. The catheter was then fixed to the skin. Intraoperatively, the position of the tip of central venous catheter was confirmed by the operating surgeon, either by palpation of the tip or by visualization when right atrium was opened. All data were tabulated and analyzed systematically. The patients, were divided according to the height $(\mathrm{H})$ into two groups of $<100 \mathrm{~cm}$ and $>100 \mathrm{~cm}$. All data were expressed as Mean \pm SD. The demographic data were analyzed using Chi square test. A P-value $<0.05$ was considered significant. Pearson's correlation coefficient was calculated to categorize any relation between height, weight or body surface area (BSA) and the depth of insertion required to locate correct positioning of central venous catheter. Plots of

Table 2. Depth of Central venous catheterization

\begin{tabular}{|c|c|c|c|c|c|}
\hline & & Weight (kg) & Height (cm) & BSA & Depth (cm) \\
\hline \multirow{4}{*}{$\begin{array}{l}\text { Height }<100 \\
N=44\end{array}$} & Mean \pm SD & $7.34 \pm 3.29$ & $72.08 \pm 13.75$ & $0.38 \pm 0.12$ & \multirow{4}{*}{$6.0 \pm 1.1$} \\
\hline & $\mathrm{R}$ & $0.37 *$ & $0.51 * *$ & $0.45^{*}$ & \\
\hline & Significance & 0.021 & 0.001 & 0.017 & \\
\hline & Range & $3-19$ & $49-97$ & $0.2-0.64$ & \\
\hline \multirow{4}{*}{$\begin{array}{l}\text { Height }>100 \\
N=25\end{array}$} & Mean $\pm \mathrm{SD}$ & $17.56 \pm 6.99$ & $115.92 \pm 13.96$ & $0.75 \pm 0.19$ & \multirow[t]{4}{*}{$7.52 \pm 1.64$} \\
\hline & $\mathrm{R}$ & $0.70 * *$ & $0.54 * *$ & $0.63 * *$ & \\
\hline & Significance & 0.000 & 0.008 & 0.001 & \\
\hline & Range & $12-46$ & $100-151$ & $0.5-1.41$ & \\
\hline
\end{tabular}

${ }^{* *}$ Correlation is significant at 0.01 level (2-tailed)

* Correlation is significant at 0.05 level (2-tailed)

$r$ Pearson correlation coefficient of Depth with Weight, Height and BSA 


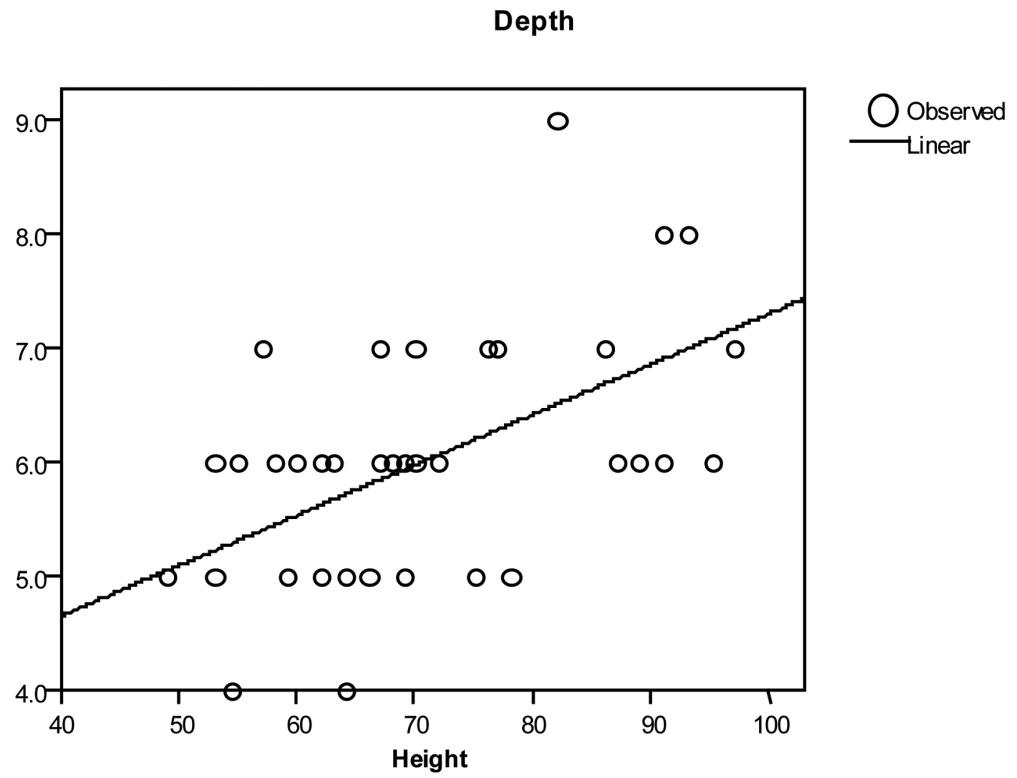

Figure 1. Depth versus height in Paediatric $<100 \mathrm{~cm}\left(\mathrm{r}^{2}=0.270 \mathrm{P}=0.001\right)$

distance were made and regression lines and equations were calculated by using SPSS 14.0 (SPSS, Chicago, IL). BlantAltman analysis of data was done to compare the old existing formulae with our derived formulae.

\section{Results}

A total of 69 patients were included in the study. There was no significant gender based differences among different groups $(\mathrm{P}=0.087)$ (Table 1$)$. As height had significant correlation at 0.05 level, further analysis was restricted to height (Table 2). Simple formulae were developed to predict placement of CVC through the right IJV. These were developed by plotting a straight line on the graph of patient's height versus depth and then deriving a simple formula describing that line. (Fig. 1, 2)

For right IJV cannulation:

Paediatric patients $<100 \mathrm{~cm}$ : Depth of insertion $(\mathrm{cm})=$ (Height in $\mathrm{cm} / 25)+2.5$

\section{Depth}

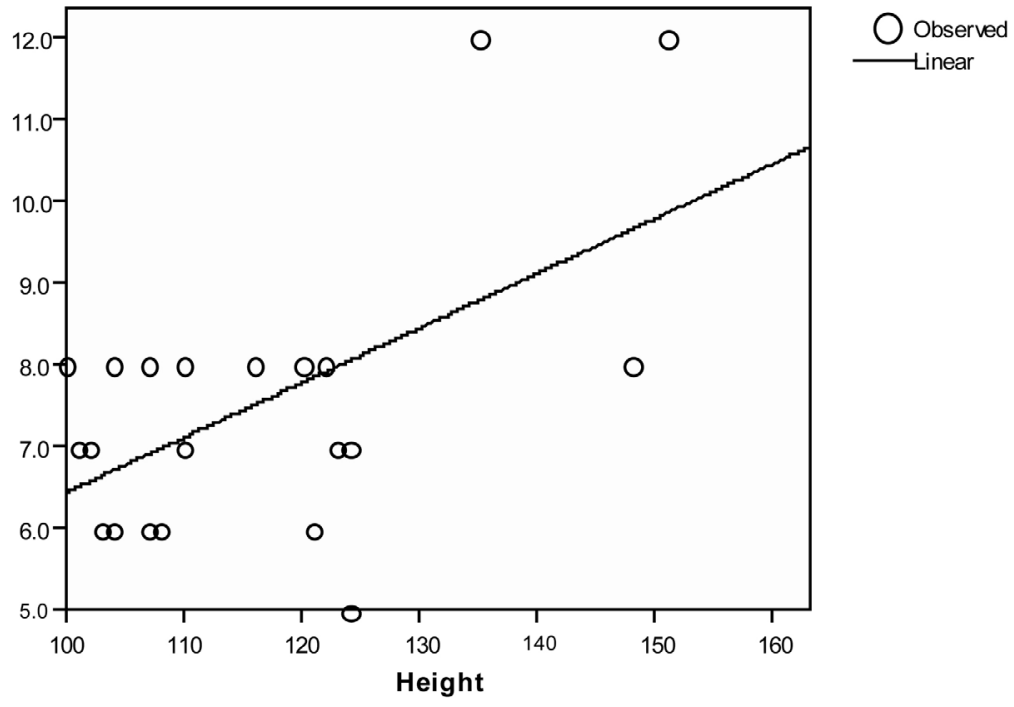

Figure 2. Bland Altman for paediatric $<100 \mathrm{~cm}$ group 


\section{Bland-Altman of Data}

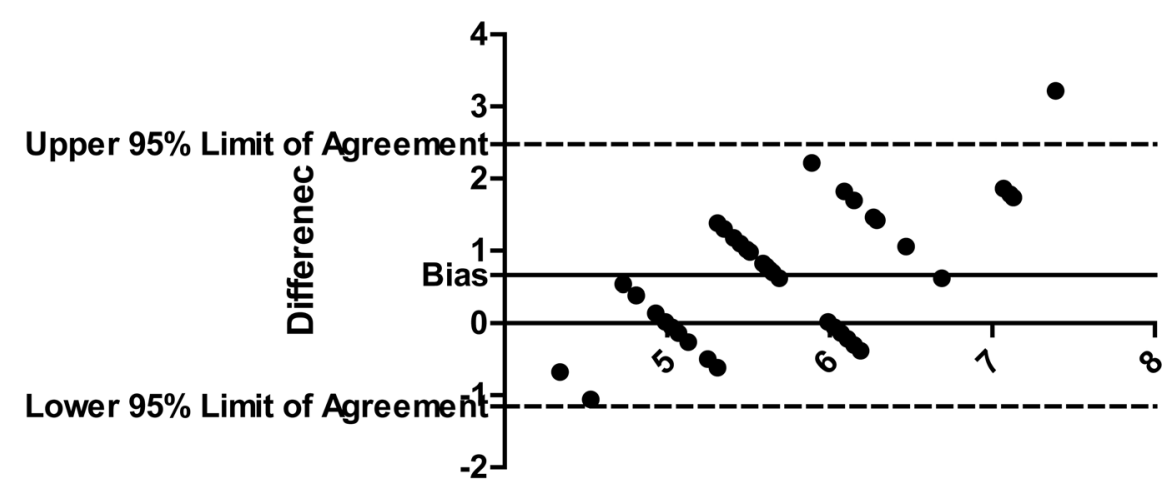

Average

Figure 3. Depth versus height in Paediatric $>100 \mathrm{~cm}$

Paediatric patients $>100 \mathrm{~cm}$ : Depth of insertion $(\mathrm{cm})=$ (Height in $\mathrm{cm} / 10)-4$

After applying Blant Altman (Fig. 3, 4) for the data in paediatric patients $>100 \mathrm{~cm}$, the bias i.e. average difference between the two sets of readings) was very small with new formula $(-0.072$; the depth measured with new formula was $0.07 \mathrm{~cm}$ more as compared with existing) as compared with existing formula (bias was -2.07; the formula measures 2.07 $\mathrm{cm}$ more than existing). Similarly, the width of $95 \%$ Confidence interval with new formula was less as compared with the width of $95 \%$ Confidence interval with existing formula.

However, in paediatric patients $<100 \mathrm{~cm}$ group, the bias was small with existing formula (-0.16; the depth measured with existing formula is $0.16 \mathrm{~cm}$ more as compared with original) as compared with new formula (bias is 0.66 ; the formula measures $0.66 \mathrm{~cm}$ less than existing). Similarly, the width of $95 \%$ Confidence interval with new formula is less as compared with the width of $95 \%$ CI with existing formula.

\section{Discussion}

The CVC is recommended to be placed in the distal superior vena cava (SVC) i.e., outside of the cardiac chamber.

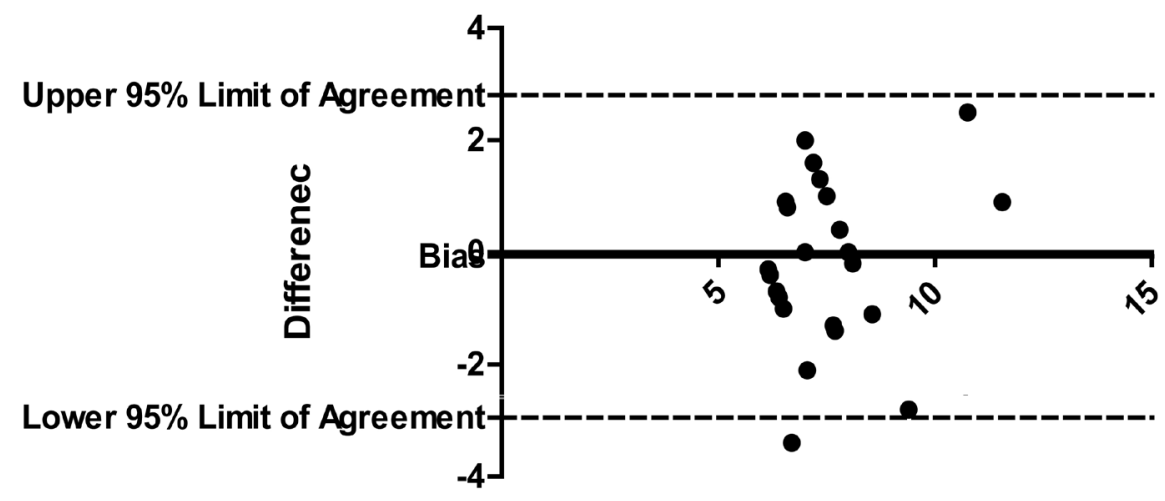

Average

\section{Bland-Altman of Data for Pead $>\mathbf{1 0 0}$}

Figure 4. Bland Altman for paediatric $>100 \mathrm{~cm}$ group 
Incorrect positioning in the RA or ventricle and misplacement in the lateral branches of the superior vena cava are the most common types of catheter misplacement. They can be avoided by correctly judging catheter length, using a gentle technique when advancing the catheter and positioning the patient in a way that facilitates access to the vein. Studies conducted over the past several years have proven that the use of an intracardiac ECG may obviate the need for routine x-rays to verify CVC placement. In 1949, Hellerstein took his previous experience with ECG leads and applied them in a unique way for directly recording atrial potentials Hellerstein's method differed from that of his colleagues who used metal wires for recording the signals, whilst he used a CVC filled with saline solution. The signal was captured at the catheter hub through a self-made adaptor and transferred to an ECG monitor. In his landmark publication, he examined the recording of both the saline solution and through a metal wire. As soon as the catheter tip reaches the sinoatrial node, there is an abrupt increase in height of the $\mathrm{P}$ wave $[1,2]$. When the tip enters into the RA, a height of the P wave potential is reached. The intracardiac ECG should be recorded when the wire and catheter are in this position. A disposable electrode cable is available in the catheter sets, so that the signal can be recorded directly from the metallic guide wire. This electrode cable is connected to a universal adaptor that allows the user to continuously switch back and forth between skin and intra-arterial lead. An electrical switching device for alternating between the skin lead and intracardiac lead is required for both the electrolyte-filled and stylet guided lead connected. Each set contains a disposable lead cable with alligator clip, which can be attached to the free end of the wire. Both the stylet and the Seldinger wire conduct the potential from the atrium and onwards through the lead cable to the ECG monitor without risk of interference. Recording the atrial ECG through the metallic guide stylet greatly simplifies handling, since all the components are present in sterile versions and are easy to connect. A maximum P-wave is derived even at the entrance to the right atrium. This explains why ECG guided CVC placement based on the largest $\mathrm{P}$ wave amplitude consistently resulted in correct positioning of the CVC tip at the transition from the superior vena cava to the right atrium. The intracardiac ECG lead produces clear and reliable information about catheter tip position for all but patients with pre-existing abnormal cardiac rhythms. Numerous clinical studies have demonstrated a high accuracy rate of over $90 \%$ for this control method. However, in our experience, the distance did not correspond according to any of the published formulae like right subclavian or jugular vein: 13 - $16 \mathrm{~cm}$ Left subclavian or jugular vein: 15 - $20 \mathrm{~cm}$ [3]. Peres and coworkers published catheterization guidelines for adult patients [4] and the working group of Andropoulos designed a formula for predicting correct CVC positioning in paediatric patients [1]. Peres devised the formula for access through the subclavian and jugular vein, while there was insufficient data available for other puncture sites. According to Peres, through Right side: External and internal jugular vein: depth is $\mathrm{H} / 10$ [4]. Andropoulos and coworkers performed a similar study in children and juveniles, but considered only access through the right subclavian and internal jugular vein. When $\mathrm{H}=100 \mathrm{~cm}$, it is $\mathrm{H} / 10-1$ and when $\mathrm{H}>100 \mathrm{~cm}$, it is $\mathrm{H} / 10-2$ [1]. The values obtained using the Andropoulos formula contradicts those of the Peres formula in patients taller than 100 $\mathrm{cm}$ and when access through the jugular vein is used. This discrepancy merits further investigation. Hence, our study was designed over a period of four months to decide on the formulae to be used for daily use.

In patients with dysrhythmia, atrial fibrillation or implanted pacemakers, the $\mathrm{P}$ wave remains unchanged in the intra-arterial ECG lead; even if the catheter is correctly withdrawn from the atrium into the vena cava [5]. There is only one single report of catheterization of the carotid artery with advancement into the right atrium, which resulted in the induction of P wave elevation. In this case, the ECG signal was interpreted as confirming correct placement although the catheter had been seriously misplaced [6]. Obligatory radiographic monitoring does not produce a clinical advantage, whereas it does increase costs unnecessarily. Constant position monitoring is required to instantly identify problems that might arise during puncture or advancement. The atrial ECG recording is therefore recommendable not only for economical reasons, but because of its high success rate [7].

The use of intracardiac ECG is a reliable, easy to learn method and can be conducted at minimal cost. The use of the ECG lead to verify CVC position has now become a standard technique in anaesthesia and intensive care medicine [8]. Physicians who have mastered this technique therefore no longer need to perform chest x-rays to monitor catheter position, since the ECG lead provides sufficiently accurate verification [8]. Routine chest x-ray after catheter placement is thereby obviated. Our formulae gave better precision as compared to previous formulae. Although this easy method is available since long, it is not utilized optimally.

\section{Conclusion}

The devised formulae can accurately predict the required depth of catheters, thereby possibly reduce the possibility of complications and need for radiographic confirmation.

\section{Acknowledgements}

Assistance with the study: None except the authors.

\section{Financial Support and Sponsorship}

None. 


\section{Conflict of Interest}

None.

\section{References}

1. Andropoulos DB, Bent ST, Skjonsby B, Stayer SA. The optimal length of insertion of central venous catheters for pediatric patients. Anesth Analg. 2001;93(4):883-886.

2. Hellerstein HK, Pritchard WH, Lewis RL. Recording of intracavity potentials through a single lumen, saline filled cardiac catheter. Proc Soc Exp Biol Med. 1949;71(1):58-60.

3. Kirby, R. R. et al.: Clinical Anesthesia Practice. 2nd: 531-540W. B. Saunders Philadelphia 2002, 2
4. Peres PW. Positioning central venous catheters--a prospective survey. Anaesth Intensive Care. 1990;18(4):536539.

5. Salmela L, Aromaa U. Verification of the position of a central venous catheter by intra-atrial ECG. When does this method fail? Acta Anaesthesiol Scand. 1993;37(1):26-28

6. Schafer M, Ciesielski K, Kuss B, Link J. [Incorrect placement of a vena cava catheter and its prevention by intra-atrial ECG]. Anaesthesist. 1988;37(1):49-51.

7. Schuster M, Panning B. Electrocardiographic guidance in placing central venous catheters. Crit Care Med. 2000;28(10):3577-3578.

8. WeiBlauer, W.: Der Cava-Katheter aus medico-legaler Sicht. Anaesthesiol. Intensivmed. Notfallmed. Schmerzther. 1998, 33: 117-118 